\title{
Update on the Clinical Application of Quantitative Computed Tomography (QCT) in Osteoporosis
}

\author{
$\mathrm{Xu} \mathrm{Li} \cdot \mathrm{Li} \mathrm{Na} \cdot$ Cheng Xiaoguang
}

Published online: 14 August 2014

(c) Springer Science+Business Media New York 2014

\begin{abstract}
Osteoporosis is becoming an increasingly important public health issue and the measurement of BMD is important for diagnosing osteoporosis as well as predicting fracture risk and evaluating therapy efficacy. DXA is currently the best established method for osteoporosis diagnosis, and, however, QCT is more sensitive to the change of BMD with the ability of measuring the volumetric BMD. With the disadvantage of the relatively higher radiation dose over DXA, the clinical application of QCT in the management of osteoporosis warrants further development.
\end{abstract}

\section{Keywords Osteoporosis · QCT · BMD}

\section{Introduction}

Osteoporosis was defined as "a disease characterized by low bone mass and microarchitectural deterioration of bone tissue, leading to enhanced bone fragility and a consequent increase in fracture risk" [1]. In addition, osteoporosisrelated fractures are one of the leading causes of significant morbidity and disability in elderly patients and increase the

This article is part of Topical Collection on Highlights of Musculoskeletal Imaging in China.

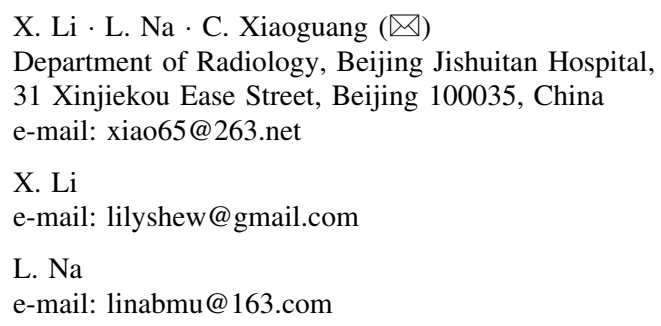

economic burden on the health care system. Osteoporosis is a kind of skeletal disorder characterized by compromised bone strength predisposing a person to increased risk of fracture [2]. Bone strength reflects the integration of bone mineral density (BMD) and quality [2]. BMD is expressed as grams of mineral mass per area or volume. The measurement of BMD is important for diagnosing osteoporosis as well as predicting fracture risk and evaluating therapy efficacy. Currently, as a technique with high precision and low radiation dose, dual X-ray absorptiometry (DXA) measurements of BMD have been universally adopted as a standard to define osteoporosis. However, DXA has some pertinent disadvantages that need to be considered: $(a)$ It is a two-dimensional (2D) measurement, which only measures density/area (in grams per square centimeter). (b) Spine and hip DXA are also sensitive to degenerative changes, and individuals with substantial degenerative disease will have increased areal density, which will suggest a lower fracture risk than actually present [3•]. Recently, along with the development of multidetector CT (MDCT) scan technique, calibration phantom, and analysis software, quantitative computed tomography (QCT) BMD measurement is attracting more attention.

\section{QCT BMD Measurement Technique}

In the late 1970s and early 1980s, QCT BMD measurement was clinically practiced [4]. To perform QCT, a standard CT scanner with an external bone mineral calibration phantom underneath the patient is used and density values measured in Hounsfield units are transformed into BMD measured in milligrams hydroxyapatite per cubic centimeter using the phantom. Pre-scan calibration and consistent parameters of scan and reconstruction are 
required to make the results comparable and accurate. QCT has been found with good precision and reproducibility by a number of clinical studies performed in China $[5 \cdot, 6]$.

While use of QCT has centered on 2D characterization of vertebral trabecular bone, there is interest in developing three-dimensional (3D) or volumetric quantitative computed tomography (vQCT) techniques both to improve spinal measurements as well as to extend QCT assessments to the proximal femur. Typically, the region of interest (ROI) should be placed inside the vertebral bodies of L1 to L3. Intra-vertebral variation in BMD measured by QCT was detected, and therefore the ROI should be placed in the middle of the vertebral body consistently to avoid measurement error [7•]. Currently, single-slice QCT techniques are highly operator dependent, requiring careful slice positioning and angulation as well as careful ROI placement. In a volumetric approach, on the other hand, an image of the entire vertebral body is acquired, and the volumes of interest (VOIs) are determined and repositioned using the software [8].

Because of the proximal femur's complex architecture and dramatic 3D variation in its density, the 2D QCT methods widely used in the spine cannot be used to assess proximal femur. Volumetric techniques are now available to measure proximal femoral BMD [3]. These 3D techniques encompass the entire object of interest either with stacked-slice or spiral CT scans and can employ anatomic landmarks by the CT image reformatting systems to define the relevant projections. These projections are equivalent to DXA images and ROIs. The 3D QCT techniques has been validated by recent study [6].

\section{Advantages and Disadvantages of QCT}

Generally, QCT is easily available on standard clinical CT scanners using an external bone mineral reference phantom to calibrate the CT number measurements to bone-equivalent values as well as special software to measure the BMD of ROI.

QCT has some important advantages over DXA: (a) It allows true volumetric measurements of the lumbar spine and proximal femur, which are independent of the body size, (b) it provides purely trabecular bone measurements, which are more sensitive to monitoring changes with disease and therapy [9], and (c) cross-sectional studies have shown that quantitative CT BMD of the spine allows better discrimination of individuals with fragility fractures $[10$, 11]. 3D reconstructed images can be used to reflect the distribution and continuity of trabecula, and in principle, the geometric parameters obtained by QCT have higher accuracy for predicting bone strength [12-14]. In addition, it should be noted that DXA has limitations in measuring
BMD in patients with a body mass index over $25 \mathrm{~kg} / \mathrm{m}^{2}$. In obese patients, superimposed soft tissue will elevate measured BMD owing to attenuation of the X-ray beams and beam hardening artifact $[15,16]$.

Recommendations for the use of QCT instead of DXA are (a) in very small or large individuals, (b) in older individuals with expected advanced degenerative disease of the lumbar spine or morphologic abnormalities, and (c) if high sensitivity to monitor metabolic bone change is required, such as in patients treated with parathyroid hormone or corticosteroids.

Pertinent disadvantages of QCT are the relatively higher radiation dose (0.06-2.9 mSv) over DXA, but the QCT radiation dose is reasonable in comparing with other routine CT scans. When QCT is added to the routine clinical CT scans, there is no additional radiation. The QCT diagnosis of osteoporosis recommended by ISCD and ACR need more education for medical professionals.

\section{Clinical Practice of QCT}

On 2007, ISCD has developed Official Positions for the clinical use of DXA and non-DXA technologies, which point out that single-slice spinal QCT should include the vertebral bodies of L1 to L3, and 3D QCT should include L1 to L2 [17]. In addition, T12 or L3 to L4 are usually the alternative vertebral bodies in case the included one is not eligible for measurement [18].

A T score is the standard deviation of the BMD of an individual patient compared with a young, healthy reference population, matched for sex and ethnicity. A T score of less than -1 to greater than -2.5 is defined as osteopenia, while a $\mathrm{T}$ score of -2.5 or lower is defined as osteoporosis by DXA. According to World Health Organization (WHO) and International Society for Clinical Densitometry (ISCD), this definition can be used for postmenopausal women and men older than 50 years [19॰, 20]. In addition, the ISCD has introduced guidelines for DXA of premenopausal women, men younger than 50 years, and children [19•, 20]. In these populations, $\mathrm{Z}$ scores are used comparing individual BMD measurements to age-matched reference populations: A $\mathrm{Z}$ score lower than -2 is defined as "below the expected range for age." It should be noted that osteoporosis cannot be defined using DXA BMD alone in these populations.

Limited number of longitudinal scientific studies assessed how QCT predicts fragility fracture, and most of all stated that $\mathrm{T}$ scores should not be used to define osteoporosis with QCT. A T score threshold of -2.5 for QCT would identify a much higher percentage of osteoporotic subjects and has therefore never been established for clinical use. Currently, vQCT techniques are state of the art 
[21-23], and in clinical routine absolute measurements of volumetric BMD to characterize fracture risk have been used $\left(110-80 \mathrm{mg} / \mathrm{cm}^{3}=\right.$ mild increase in fracture risk, $80-50 \mathrm{mg} / \mathrm{cm}^{3}=$ moderate increase in fracture risk, and $50 \mathrm{mg} / \mathrm{cm}^{3}=$ severe increase in fracture risk). According to the suggestion by ISCD on 2007, BMD measurements by QCT can be used for diagnosing osteoporosis $\left(\right.$ BMD $>120 \mathrm{mg} / \mathrm{cm}^{3}$ : normal, BMD $=80-120 \mathrm{mg} / \mathrm{cm}^{3}$ : osteopenia, and $\mathrm{BMD}<80 \mathrm{mg} / \mathrm{cm}^{3}$ or $\mathrm{T}$ score $<-3.4$ : osteoporosis) [17].

For the reason that the WHO diagnostic standards for osteoporosis cannot be used by the technologies other than DXA, some researches compared the correlations of BMD to fractures between DXA and spinal QCT, and the equivalent $\mathrm{T}$ score can be used for diagnosis with a comparable sensitivity and specificity [24, 25]. For the reasons that various CT scanner, software for image analysis, and study populations were included, the value of equivalent $\mathrm{T}$ score $(-2.9$ to -3.8$)$ for diagnosing osteoporosis was calculated by different studies [24-27].

For the postmenopausal women, spinal trabecular BMD measured by QCT shows comparable predictive ability for vertebral fracture in comparison to DXA. By comparing the abilities of discriminating vertebral fracture from osteoporosis in postmenopausal women between QCT and DXA, 3D QCT was superior to DXA, in addition significant difference was detected. Moreover, trabecular BMD measured by spinal can be used in monitoring the BMD changes related to age, diseases, and treatments [28].

\section{Epidemiology of Osteoporosis in China}

Mean value of BMD varies among different age, genders, regions, and races. Sixty to eighty percent of peak bone mass is affected by genetic factors [29], and twenty percent is affected by environmental factors. The variation of bone mass among different races and genders can be explained by the genetic difference of skeleton structure as well as the difference of height and weight. The bone mass of men is higher than that of women in each race, and the rate of fracture is lower in men. Moreover, the bone mass is different among the population in various countries as well as various regions of the same country.

Zhao, et al. investigated 704 men of Han and Uygur ethnics living in the Xinjiang province of China and found that the change trend of bone mass measured by QCT was same between the two ethnics; nevertheless, the peak value of bone mass and the prevalence of osteoporosis were higher for the Uygur ethnic compared to the Han ethnic [30•]. The differences of peak value of BMD, age of peak $\mathrm{BMD}$, and prevalence of osteoporosis were also detected by other Chinese studies [31-33]. Some researches pointed out that polymorphism of gene (i.e., leptin receptor Gln223Arg, PPAR $\gamma$ ) in populations correlated with BMD, which may be one of the factors affecting the peak bone mass [34, 35].

The study performed by Cheng, et al. included eight thousand of samples from 6 areas (Beijing, Guangzhou, Chengdu, Shanghai, Nanjing, and Jiaxing) in China and did not detected significant difference of spinal or femoral BMD among those 6 areas although there was a difference of body figure [36]. The prevalence of osteoporosis, defined as a BMD of $<$ or $=-2.5$ SDs from YA values established in that study, in female subjects of age $50 \mathrm{yr}$ or older, was $28 \%$ for the spine, $15 \%$ for any femur site, and $31 \%$ for any spine or femur site [36]. However, another study of multiple centers by $\mathrm{Li}$, et al. showed that the prevalence of osteoporosis in the women older than 40 years was significantly different $(24.5 \%-11.8 \%)$ among the 5 areas (Jilin, Shanghai, Sichuan, Guangzhou, and Beijing) in China, and in addition, the prevalence of osteoporosis in old men was lower in comparison to old women (11.5\% vs. $19.9 \%$ ) [37]. Ma, et al. investigated the prevalence of osteoporotic spinal fracture in the people of middle and old age in Chengdu and found that the prevalence of spinal fracture increased along with the increase of age, and in addition, women $(17.3 \%)$ and the country dwellers $(19.0 \%)$ presented a higher prevalence compared to men $(15.0 \%)$ and the city dweller $(13.3 \%)$ [38].

\section{Conclusions}

BMD measurement is the critical method for screening and diagnosing osteoporosis as well as monitoring the effects of treatments. DXA and QCT are the most commonly used techniques for BMD measurement. DXA is currently the best established method for osteoporosis diagnosis in vivo because of its easy availability and low radiation dose. As the only technique to measure the volumetric BMD currently, QCT is more sensitive to the change of BMD in the progress of osteoporosis diagnosis and treatment. The clinical application of QCT in the management of osteoporosis warrants further development.

\section{Compliance with Ethics Guidelines}

Conflict of Interest Dr. $\mathrm{Xu} \mathrm{Li}$ and $\mathrm{Dr}$. $\mathrm{Li} \mathrm{Na}$ each declare no potential conflicts of interest. Dr. Cheng Xiaoguang is a section editor for Current Radiology Reports.

Human and Animal Rights and Informed Consent This article does not contain any studies with human or animal subjects performed by any of the authors. 


\section{References}

Papers of particular interest, published recently, have been highlighted as:

- Of importance

1. Kanis JA, Melton LJ 3rd, Christiansen C, Johnston CC, Khaltaev N. The diagnosis of osteoporosis. J Bone Miner Res. 1994; 9(8):1137-41.

2. NIH. NIH Consensus Development Panel on Osteoporosis Prevention, Diagnosis, and Therapy, March 7-29, 2000: highlights of the conference. South Med J. Jun 2001;94(6):569-573.

3. - Link TM. Osteoporosis imaging: state of the art and advanced imaging. Radiology. Apr 2012;263(1):3-17. This article describes novel techniques to study bone quality and how standard imaging techniques should be used to diagnose prevalent osteoporotic fractures.

4. Genant HK, Boyd D. Quantitative bone mineral analysis using dual energy computed tomography. Invest Radiol. Nov-Dec. 1977;12(6):545-51.

5. - Chen XS, Cheng XG, Peng JH, Li BQ, Su JS, Dai CL. Assessment of precision and accuracy of spinal QCT in a multicentre clinical research with an European spine phantom. Chinese journal of medical imaging. 2011;19(12):912-917. The precision and accuracy of QCT is critical for its effects in measuring BMD. This article provides the precision and accuracy of QCT in a multi-centre research.

6. Kong LY, Ma YM, Wang QQ, et al. Reproducibility of hip bone mineral density measurement by quantitative CT and its consistency with DXA bone mineral density. Chin J Osteoporosis \& Bone Miner Res. 2013;6(4):334-9.

7. - Li BQ, Sun JL, Zhang X, et al. The intravertebral BMD variation measured by QCT. Chinese journal of medical imaging. 2011;19(12):893-895. The results of BMD measured by QCT may be affected by the difference of ROI placement. This work offers the intravertebral variation in BMD measured by QCT and how to avoid the measurement variation.

8. Lang T, Augat P, Majumdar S, Ouyang X, Genant HK. Noninvasive assessment of bone density and structure using computed tomography and magnetic resonance. Bone. 1998;22(5 Suppl):149S-53S.

9. Black DM, Greenspan SL, Ensrud KE, et al. The effects of parathyroid hormone and alendronate alone or in combination in postmenopausal osteoporosis. N Engl J Med. 2003;349(13):1207-15.

10. Bergot C, Laval-Jeantet AM, Hutchinson K, Dautraix I, Caulin F, Genant HK. A comparison of spinal quantitative computed tomography with dual energy X-ray absorptiometry in European women with vertebral and nonvertebral fractures. Calcif Tissue Int. 2001;68(2):74-82.

11. Yu W, Gluer CC, Grampp S, et al. Spinal bone mineral assessment in postmenopausal women: a comparison between dual $\mathrm{X}$-ray absorptiometry and quantitative computed tomography. Osteoporos Int. 1995;5(6):433-9.

12. Biggemann M, Hilweg D, Brinckmann P. Prediction of the compressive strength of vertebral bodies of the lumbar spine by quantitative computed tomography. Skeletal Radiol. 1988;17(4):264-9.

13. Mosekilde L, Bentzen SM, Ortoft G, Jorgensen J. The predictive value of quantitative computed tomography for vertebral body compressive strength and ash density. Bone. 1989;10(6):465-70.

14. Eriksson SA, Isberg BO, Lindgren JU. Prediction of vertebral strength by dual photon absorptiometry and quantitative computed tomography. Calcif Tissue Int. 1989;44(4):243-50.

15. Binkley N, Krueger D, Vallarta-Ast N. An overlying fat panniculus affects femur bone mass measurement. J Clin Densitom. Fall. 2003;6(3):199-204.
16. Tothill P, Hannan WJ, Cowen S, Freeman CP. Anomalies in the measurement of changes in total-body bone mineral by dualenergy X-ray absorptiometry during weight change. J Bone Miner Res. 1997;12(11):1908-21.

17. Engelke K, Adams JE, Armbrecht G, et al. Clinical use of quantitative computed tomography and peripheral quantitative computed tomography in the management of osteoporosis in adults: the 2007 ISCD Official Positions. J Clin Densitom. JanMar. 2008;11(1):123-62.

18. Steiger P, Block JE, Steiger S, et al. Spinal bone mineral density measured with quantitative CT: effect of region of interest, vertebral level, and technique. Radiology. 1990;175(2):537-43.

19. • Lewiecki EM, Baim S, Langman CB, Bilezikian JP. The official positions of the International Society for Clinical Densitometry: perceptions and commentary. $J$ Clin Densitom. Jul-Sep 2009;12(3):267-271. BMD measured by DXA is currently the standard technique for diagnosing osteoporosis. This article provides the definition of osteoporosis by means of DXA.

20. Lewiecki EM, Gordon CM, Baim S, et al. Special report on the 2007 adult and pediatric Position Development Conferences of the International Society for Clinical Densitometry. Osteoporos Int. 2008;19(10):1369-78.

21. Bousson V, Le Bras A, Roqueplan F, et al. Volumetric quantitative computed tomography of the proximal femur: relationships linking geometric and densitometric variables to bone strength. Role for compact bone. Osteoporos Int. 2006;17(6):855-64.

22. Farhat GN, Cauley JA, Matthews KA, et al. Volumetric BMD and vascular calcification in middle-aged women: the Study of Women's Health Across the Nation. J Bone Miner Res. 2006;21(12):1839-46.

23. Farhat GN, Strotmeyer ES, Newman AB, et al. Volumetric and areal bone mineral density measures are associated with cardiovascular disease in older men and women: the health, aging, and body composition study. Calcif Tissue Int. 2006;79(2):102-11.

24. Cann CE, Genant HK, Kolb FO, Ettinger B. Quantitative computed tomography for prediction of vertebral fracture risk. Bone. 1985;6(1):1-7.

25. Lafferty FW, Rowland DY. Correlations of dual-energy X-ray absorptiometry, quantitative computed tomography, and single photon absorptiometry with spinal and non-spinal fractures. Osteoporos Int. 1996;6(5):407-15.

26. Odvina CV, Wergedal JE, Libanati CR, Schulz EE, Baylink DJ. Relationship between trabecular vertebral body density and fractures: a quantitative definition of spinal osteoporosis. Metabolism. 1988;37(3):221-8.

27. Felsenberg D, Gowin W. Bone densitometry by dual energy methods. Radiologe. 1999;39(3):186-93.

28. Cai ZY, Wang LY, Wu SY, Lan J, Li JX. Evaluation of bone mineral density measurement of lumbar vertebrae by volumetric quantitative $\mathrm{CT}$ in postmenopausal women. Chinese journal of radiology. 2009;43(2):119-25.

29. Guglielmi G, de Terlizzi F, Torrente I, Mingarelli R, Dallapiccola B. Quantitative ultrasound of the hand phalanges in a cohort of monozygotic twins: influence of genetic and environmental factors. Skeletal Radiol. 2005;34(11):727-35.

30. - Zhao Y, Li H, Liu WY, Cheng XG, Ma H, Wang HT. Differences of bone mineral density in healthy Han and Uygur males in Xinjiang measured by quantitative CT. Chinese journal of medical imaging. 2011;19(12):899-902. The collection of BMD values by QCT in populations of different genders, races, ages and areas is important for the construction of reference database and the diagnosis definition for osteoporosis. This article provides the status of BMD and the BMD change with age by means of QCT in a large study sample.

31. Gao GY, Liu PH, Ma ZQ, Jiang FB, Wu DY, Yang L. Investigation on bone mineral density in healthy Dai and Han 
nationalities in XiShuangBanNa. Chinese journal of osteoporosis. 2008;12(4).

32. Zhang XL, Yan HQ, Hao YQ. Ethnic difference in peak bone mass. Chinese journal of osteoporosis. 2008;14(6).

33. Guo H, He XY, Zhang TL, Kong DW, Li BY. Investigation results and variation analysis of forearm bone mineral density in healthy Han and Uygur people in Xinjiang. Chinese journal of osteoporosis. 2012;18(2).

34. Qiao J, Meng XW, Xing XP, et al. Bone mineral density and leptin receptor polymorphism Gln223 Arg in Han women in Beijing. Chinese journal of internal medicine. 2004;43(4):276-9.

35. Ogawa S, Urano T, Hosoi T, et al. Association of bone mineral density with a polymorphism of the peroxisome proliferatoractivated receptor gamma gene: PPARgamma expression in osteoblasts. Biochem Biophys Res Commun. 1999;260(1):122-6.
36. Cheng XG, Yang DZ, Zhou Q, et al. Age-related bone mineral density, bone loss rate, prevalence of osteoporosis, and reference database of women at multiple centers in China. J Clin Densitom. Jul-Sep. 2007;10(3):276-84.

37. Li NH, Ou PZ, Zhu HM, Yang DZ, Zheng PR. Prevalence rate of osteoporosis in the mid - aged and elderly in selected parts of China. Chin Med J (Engl). 2002;115(5):773-5.

38. Ma JF, Wang WZ, Jiang JJ, Chen L, Yang DZ. Investigation on related factors of osteoporotic vertebral compression fracture among 1080 middle-aged and aged people having health examination in Chengdu. Chinese journal of clinical rehabilitation. 2004;8(20):3922-3. 